\title{
Arsenic trioxide potentiates sensitivity of multiple myeloma cells to lenalidomide by upregulating cereblon expression levels
}

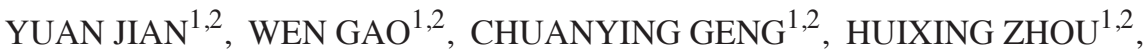 \\ YUN LENG ${ }^{1,2}$, YANCHEN LI ${ }^{1,2}$ and WENMING CHEN ${ }^{1,2}$ \\ ${ }^{1}$ Department of Hematology; ${ }^{2}$ Multiple Myeloma Research Center of Beijing, Beijing \\ Chao-Yang Hospital, Capital Medical University, Beijing 100020, P.R. China
}

Received December 4, 2015; Accepted May 11, 2017

DOI: $10.3892 / 01.2017 .6502$

\begin{abstract}
The mechanism of the anti-myeloma effect of the immunomodulatory drug lenalidomide relies upon the binding of lenalidomide or an analogue to cereblon (CRBN) ubiquitin ligase, which inhibits it and results in the degradation of Ikarosfamily zinc finger proteins 1 and 3 (IKZF1 and IKZF3). To determine whether the traditional Chinese medicine arsenic trioxide, could potentiate sensitivity of multiple myeloma (MM) cells to lenalidomide and identify the mechanism by which this happens, the present study investigated how arsenic trioxide affected CRBN on MM cell lines and examined the anti-myeloma effect and mechanism in the combination of arsenic trioxide and lenalidomide. The present study revealed that arsenic trioxide upregulates the transcription and protein levels of CRBN, the anti-myeloma target of lenalidomide, thus potentiating the sensitivity of multiple myeloma cells to lenalidomide and enhancing the lenalidomide-dependent degradation of IKZF1 and IKZF3. The results of the present study indicate that clinical trials of this combination therapy could take place within the near future, with the aim of improving MM patient outcome.
\end{abstract}

\section{Introduction}

Multiple myeloma (MM) is a clonal plasma cell malignancy that leads to an accumulation of plasma cells in the bone marrow. Although the treatment response and survival rates of MM patients have markedly improved over the last decade owing to the broad use of novel agents, the majority of patients will eventually develop resistant disease (1). As such, novel therapeutic options are required by MM patients. Immunomodulatory drugs (IMiDs), including lenalidomide

Correspondence to: Professor Wenming Chen, Department of Hematology, Beijing Chao-Yang Hospital, Capital Medical University, 8 Gongti South Road, Chaoyang, Beijing 100020, P.R. China

E-mail: xybxx@ccmu.edu.cn

Key words: arsenic trioxide, multiple myeloma, cereblon, lenalidomide, synergistic effect and pomalidomide, are thalidomide analogues that have efficacy in several hematological malignancies, including MM $(2,3)$. Nevertheless, only $30 \%$ of patients respond to these drugs when used as single agent, and the majority of patients will develop drug resistance (4).

Although several mechanisms of action have been proposed to explain the direct and indirect anti-myeloma effect of IMiDs, such as antiangiogenic, pro-apoptotic, antiproliferative and immunomodulatory effects (5), the precise molecular mechanisms and targets through which IMiDs exert their effects remained unclear until 2010, when Ito et al (6) identified cereblon (CRBN) as a primary target of thalidomide teratogenicity by generating an E3 ubiquitin ligase complex with DDB1, CUL4 and Roc1. A further study by Zhu et al (7) confirmed that CRBN was also required for the anti-myeloma activity of lenalidomide and pomalidomide.

Arsenic trioxide is a traditional Chinese medicine that has been used therapeutically for 2400 years (8); it is an effective treatment for acute promyelocytic leukemia (APL) and can induce complete remission in patients $(9,10)$. Arsenic trioxide affects numerous intracellular signal transduction pathways and causes multiple alterations to cellular function, resulting in the induction of apoptosis, the inhibition of growth and angiogenesis and the promotion of differentiation in a wide variety of malignancies, including MM (11). Thus, arsenic trioxide may be an attractive therapeutic tool for overcoming the resistance of myeloma cells to multiple agents.

To determine whether arsenic trioxide could potentiate the sensitivity of multiple myeloma cells to lenalidomide and to specify the mechanism by which this happens, the present study investigated how arsenic trioxide affected CRBN in MM U266 and RPMI8226 cell lines. Arsenic trioxide upregulates the transcription and protein level of CRBN, the anti-myeloma target of lenalidomide, and thus potentiates sensitivity of multiple myeloma cells to lenalidomide, which sets the stage for potential clinical trials of combination therapy to improve patient outcome in MM.

\section{Materials and methods}

Reagents. Lenalidomide (CC-5013) was purchased from Selleck Chemicals (Houston, TX, USA) and dissolved in dimethyl sulfoxide to $10 \mathrm{mM}$. Arsenic trioxide was obtained 
from Heilongjiang Harbin Medical University Pharmaceutical Co., Ltd. (Harbin, China) at a concentration of $5 \mathrm{mM}$. Drugs were diluted in culture medium prior to use at the indicated working concentration.

Cell culture, and assessment of cell viability, apoptosis and cell cycle. Human MM U266 and RPMI8226 cell lines (American Type Culture Collection, Manassas, VA, USA) were cultured in RPMI 1640 medium supplemented with $10 \%$ fetal bovine serum, $100 \mathrm{U} / \mathrm{ml}$ penicillin, $100 \mu \mathrm{g} / \mathrm{ml}$ streptomycin, and $2 \mathrm{mM}$ L-glutamine (all from Thermo Fisher Scientific, Inc., Waltham, MA, USA) at $37^{\circ} \mathrm{C}$ in a $5 \% \mathrm{CO}_{2}$ humidified atmosphere. Analysis of cell viability was performed using Cell Counting Kit-8 (CCK-8; Beijing Solarbio Science \& Technology Co., Ltd., Beijing, China), according to the manufacturer's instructions. The combination index (CI) of arsenic trioxide and lenalidomide was calculated using CompuSyn 1.0 software (ComboSyn, Inc., Paramus, NJ, USA) based on the method outlined by Chou (12) to determine the existence of synergism; a CI value less than 1.0 indicates synergism and a value less than 0.1 indicates strong synergism. The position of cells in the cell cycle was analyzed using propidium iodide staining by Cell Cycle and Apoptosis Analysis kit (Beyotime Institute of Biotechnology, Jiangsu, China), according to the manufacturer's instructions, followed by analysis on BD FACSCanto II (BD Biosciences, San Jose, CA, USA). Apoptosis was quantified using the Annexin V-FITC/Propidium Iodide Apoptosis Detection kit (Nanjing KeyGen Biotech Co., Ltd., Nanjing, China), according to the manufacturer's instructions, followed by analysis using a BD FACSCanto II flow cytometer (BD Biosciences, San Jose, CA, USA).

Western blotting. Cells were harvested after $24 \mathrm{~h}$ incubation at $37^{\circ} \mathrm{C}$ and lysed in radioimmunoprecipitation lysis buffer (Beyotime Institute of Biotechnology) with $1 \%$ phenylmethanesulfonyl fluoride (Pierce; Thermo Fisher Scientific, Inc.), according to the manufacturer's instructions. The BCA protein assay kit (Nanjing KeyGen Biotech Co., Ltd.) was used to determine total protein concentration. Proteins were separated on a $10 \%$ Tris- $\mathrm{HCl}$ polyacrylamide gel and transferred to a polyvinylidene fluoride membrane (Bio-Rad Laboratories, Inc., Hercules, CA, USA). The membrane was blocked for $1 \mathrm{~h}$ in PBS with $0.1 \%$ Tween-20 and 5\% non-fat dry milk, then incubated overnight at $4^{\circ} \mathrm{C}$ with antibodies against CRBN (catalog no. H6-NBP1-91810; dilution, 1:1,000; rabbit antihuman; Novus, Littleton, CO, USA), Ikaros family zinc finger proteins 1 (IKZF1; catalog no. 23210002; dilution, 1:5,000; rabbit anti-human; Novus), IKZF3 (catalog no. ab139408; dilution, 1:10,000; rabbit anti-human; Abcam, Cambridge, UK), and $\beta$-actin (catalog no. NB600-501; dilution, 1:1,500; mouse anti-human; Novus), followed by washing three times with Tris-buffered saline-Tween (Beijing Solarbio Science and Technology Co., Ltd., Beijing, China). Membranes were subsequently incubated with IRDye 680-conjugated goat antirabbit IgG (catalog no. 926-32221; dilution, 1:10,000; LI-COR Biosciences, Lincoln, NE, USA) and IRDye 680-conjugated goat anti-mouse IgG (catalog no. 926-32220; dilution, 1:15,000; LI-COR Biosciences) for $1 \mathrm{~h}$ at room temperature. Antigen-antibody complexes were detected using the Odyssey
Two-Color Infrared Imaging system (LI-COR Biosciences). Densitometry was conducted using Image $\mathbf{J}$ software (version 2.1.4.7; National Institutes of Health, Bethesda, MD, USA) to obtain semi-quantitative data of each band.

RNA extraction and reverse transcription-quantitative polymerase chain reaction ( $R T-q P C R)$. Total RNA extraction from different cells was performed using the RNAprep Pure Cell/Bacteria kit (TIANGEN Biotech Co., Ltd., Beijing, China), according to the manufacturer's protocol. The RNA concentration was measured by absorbance of ultraviolet light at a wavelength of $260 \mathrm{~nm}$, using the spectrophotometer Nanodrop 2000 (Thermo Fisher Scientific, Inc.), and the integrity of the extracted total RNA was detected by $1 \%$ agarose gel electrophoresis. Reverse transcription was performed using a TIANScript RT kit (TIANGEN Biotech Co., Ltd.) with oligodT primer, according to the manufacturer's protocol. qPCR was performed using SYBRGreen Real MasterMix (TIANGEN Biotech Co., Ltd.), according to the manufacturer's instructions. qPCR was conducted using RealMasterMix/SYBR solution (9 $\mu \mathrm{l}$, including 20X Mix SYBR solution $1 \mu \mathrm{l}$ and 2.5X RealMasterMix $8 \mu \mathrm{l}$; TIANGEN Biotech Co., Ltd.), primer $(1 \mu \mathrm{l}, 5 \mathrm{pmol} / \mathrm{l})$, template DNA $(2 \mu \mathrm{l})$, and sterile water (7 $\mu \mathrm{l})$. The reaction conditions for $\mathrm{qPCR}$ were $95^{\circ} \mathrm{C}$ for $2 \mathrm{~min}$, followed by 40 cycles of $95^{\circ} \mathrm{C}$ for $15 \mathrm{sec}, 60^{\circ} \mathrm{C}$ for $30 \mathrm{sec}$ and $68^{\circ} \mathrm{C}$ for $50 \mathrm{sec}$. All reactions were performed in triplicate. Primers for CRBN and GAPDH were obtained from Sangon Biotech Co., Ltd. (Shanghai, China). Primer sequences for the analyzed genes were as follows: CRBN forward, 5'-CAG TCT GCC GAC ATC ACA TAC-3' and reverse, 5'-GCA CCA TAC TGA CTT CTT GAGGG-3'; GAPDH forward, 5'-AAG GTC GGA GTC AAC GGATT-3' and reverse, 5'-CTC CTG GAA GAT GGT GATGG-3'. Expression levels were calculated using the $2^{-\Delta \Delta C q}$ method (13).

Statistical analysis. All in vitro experiments were performed in triplicate and repeated at least three times; a representative experiment was selected for figures. Statistical significance of differences observed in drug-treated vs. control cultures were determined using Student's t-test, with $\mathrm{P}<0.05$ considered to indicate a statistically significant difference. Statistical graphs were produced using GraphPad Prism 6.0 (GraphPad Software, Inc., La Jolla, CA, USA).

\section{Results}

Low doses of arsenic trioxide upregulates CRBN expression levels in MM cell lines U266 and RPMI8226. As CRBN acts as the target of lenalidomide in MM cells, the present study investigated whether arsenic trioxide treatment results in the upregulation of CRBN. U266 and RPMI8226 cells were treated with varying concentrations of arsenic trioxide in vitro for $24 \mathrm{~h}$ and detected the expression levels of CRBN mRNA under different arsenic trioxide concentrations. As a result, an increase in CRBN mRNA level was observed within a certain arsenic trioxide concentration range (between 0.1 and $1 \mu \mathrm{M}$ ) both in U266 and in RPMI8226 (Fig. 1A and B). For U266 cells, arsenic trioxide elevated CRBN expression levels by 2.96 -fold at a treatment concentration of $0.1 \mu \mathrm{M}$, 4.31-fold at a treatment concentration of $0.5 \mu \mathrm{M}$, and 8.41-fold 


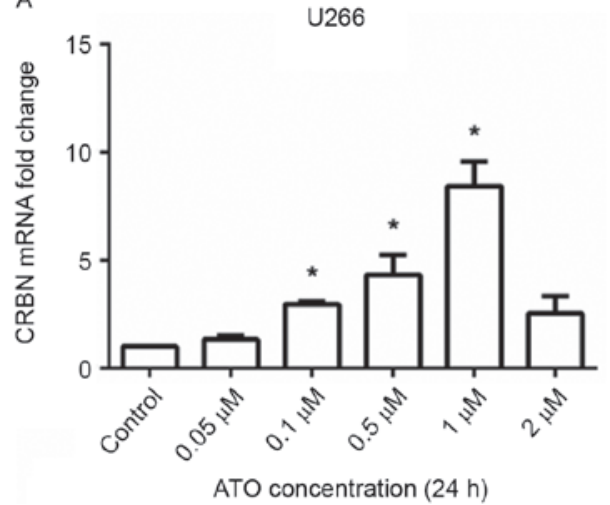

B

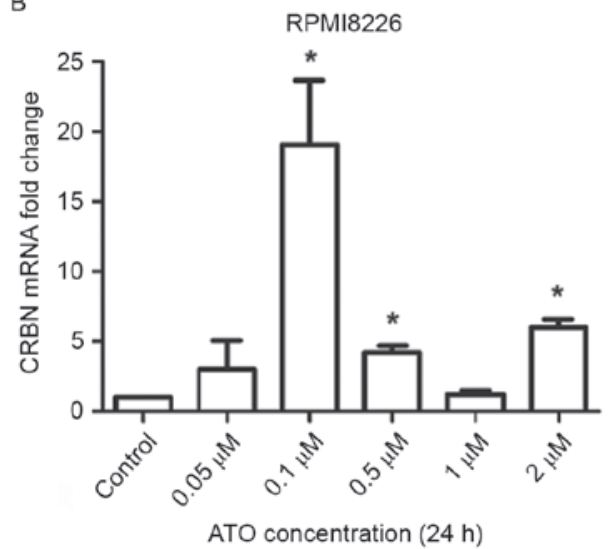

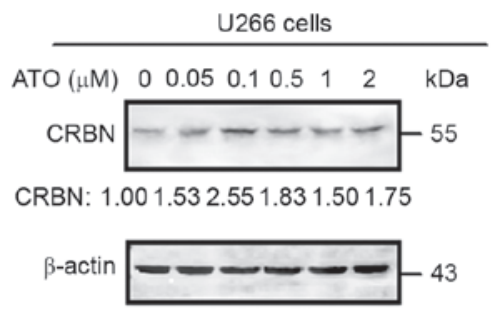

D

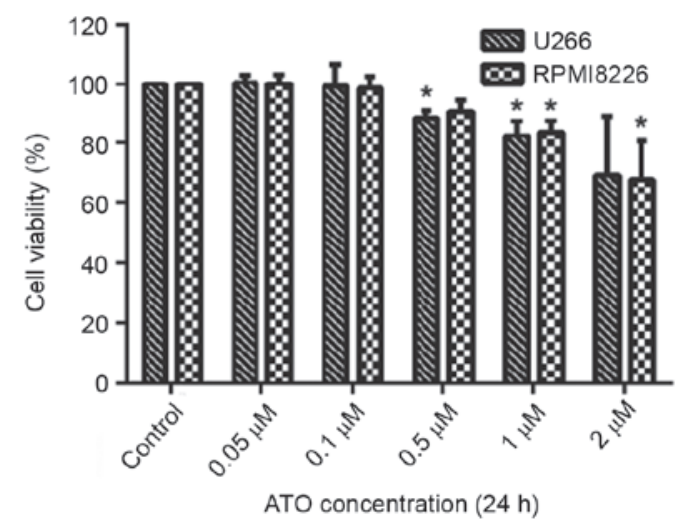

Figure 1. Low doses of arsenic trioxide increase the mRNA expression in multiple myeloma U266 and RPMI8226 cell lines. (A) Gradient doses of arsenic trioxide altered the expression of U266 CRBN mRNA expression. Longitudinal coordinates represent the relative fold change, compared to the corresponding level of GAPDH mRNA. (B) Gradient doses of arsenic trioxide differentially altered the expression of CRBN mRNA by RPMI8226 cells. (C) The effect of gradient doses of arsenic trioxide on CRBN and $\beta$-actin protein expression levels was assessed by western blot analysis. Blots for CRBN were quantified by using ImageJ software (version 2.1.4.7). Values indicate the fold-increase in expression following normalization to the loading control ( $\beta$-actin). (D) The viability inhibition of the indicated doses of arsenic trioxide in U266 and RPMI8226 cells within the same duration. Values plotted are the mean \pm standard deviation ( $\mathrm{n}=3$ ). ${ }^{*} \mathrm{P}<0.05$ (compared with control group). CRBN, cereblon; ATO, arsenic trioxide.

at a treatment concentration of $1 \mu \mathrm{M}$, compared with the control group $(\mathrm{P}<0.05)$. In RPMI8226 cells, CRBN expression levels increased 19.07-fold upon treatment with $0.1 \mu \mathrm{M}$ arsenic trioxide $(\mathrm{P}<0.05)$. The results of western blotting also demonstrated the upregulation of CRBN protein levels upon treatment with the aforementioned concentrations of arsenic trioxide, particularly at a concentration of $0.1 \mu \mathrm{M}$ (Fig. 1C). The inconsistency between the expression of CRBN mRNA and protein may be due to the difference in expression between the full length and spliced forms of mRNA upon treatment with arsenic trioxide. As the change at protein level was more important in a biological context, $0.1 \mu \mathrm{M}$ arsenic trioxide was chosen as the concentration to be used for the following experiments. The initial effective concentration of arsenic trioxide that increased CRBN mRNA and protein expression levels was $0.1 \mu \mathrm{M}$, which was much lower than the quarter-maximal inhibitory concentration $\left(\mathrm{IC}_{25}\right)$ in both cell lines $(\sim 2 \mu \mathrm{M}$ for each cell line) (Fig. 1D), indicating that arsenic trioxide increases CRBN expression levels by a different mechanism from the one through which is viability inhibited or apoptosis driven.

Combined low doses of arsenic trioxide and lenalidomide trigger synergistic anti-MM activity. As arsenic trioxide could upregulate CRBN expression levels in the MM U266 and RPMI8226 cell lines, whether pretreatment of arsenic trioxide could result in increased sensitivity to lenalidomide in these two cell lines was investigated. U266 and RPMI8226 cells were pretreated with $0.1 \mu \mathrm{M}$ arsenic trioxide for $24 \mathrm{~h}$ and lenalidomide was then added to a final concentration of $10 \mu \mathrm{M}$ for an additional $24 \mathrm{~h}$, followed by assessment for cell viability using CCK-8 assays. Arsenic trioxide and lenalidomide were used at concentrations lower than the maximal cytotoxic concentration for each cell line. A significant decrease in viability of both cell lines was observed in response to the combination therapy $(\mathrm{P}<0.05$; $\mathrm{n}=3$; Fig. $2 \mathrm{~A}$ and B). Treatment of U266 cells with low doses of arsenic trioxide $(0.1 \mu \mathrm{M})$ and lenalidomide $(10 \mu \mathrm{M})$ triggered a $16 \%$ decrease in cell viability, whereas only minimal viability inhibition was observed using either of these agents alone at these low concentrations (Fig. 2A). The same effect was also observed in RPMI8226 cells: Treatment with low doses of the two agents together $(0.1 \mu \mathrm{M}$ arsenic trioxide and $10 \mu \mathrm{M}$ lenalidomide) triggered a $17 \%$ decrease in cell viability, whereas only minimal viability inhibition was observed using either of these agents alone at these low concentrations (Fig. 2B). CI calculation confirmed the strong synergistic anti-MM activity of these two agents, with a CI value $<0.1$ in both MM-cell lines tested. These data demonstrate the synergistic anti-MM activity of arsenic trioxide plus lenalidomide. 
A

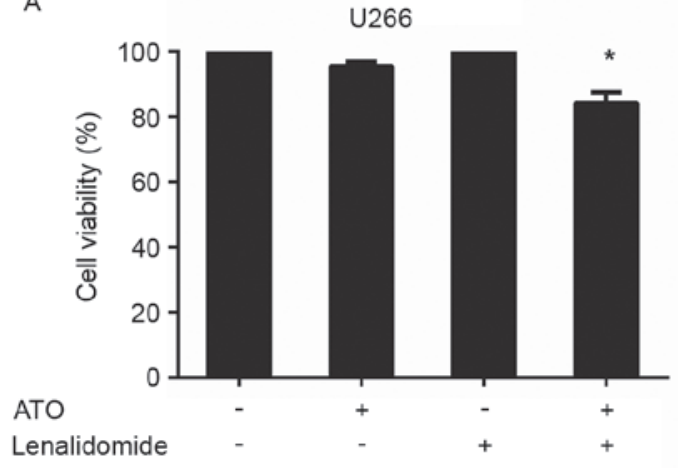

B

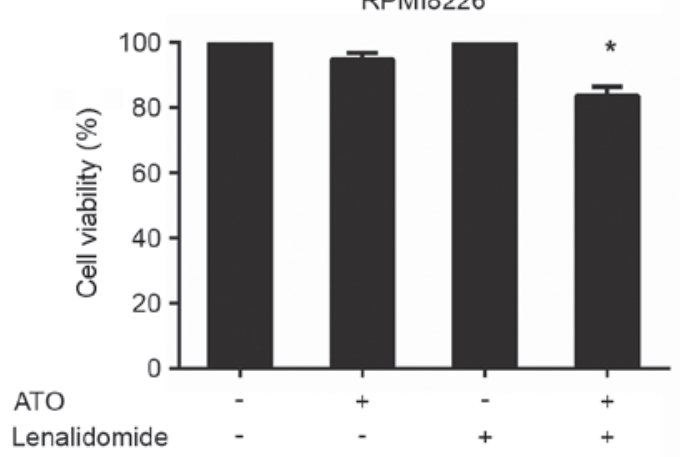

C

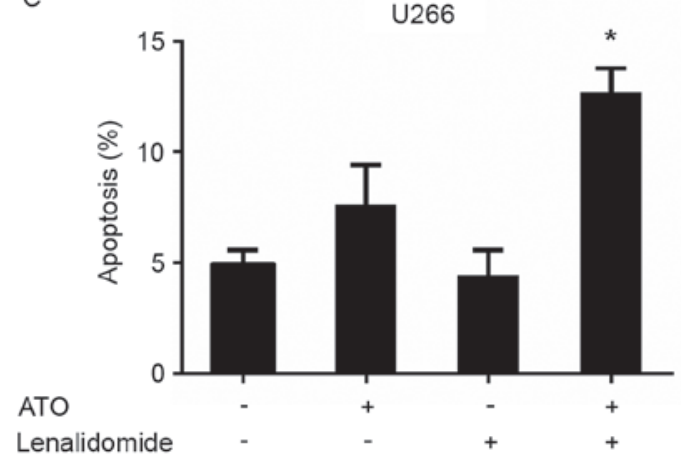

D

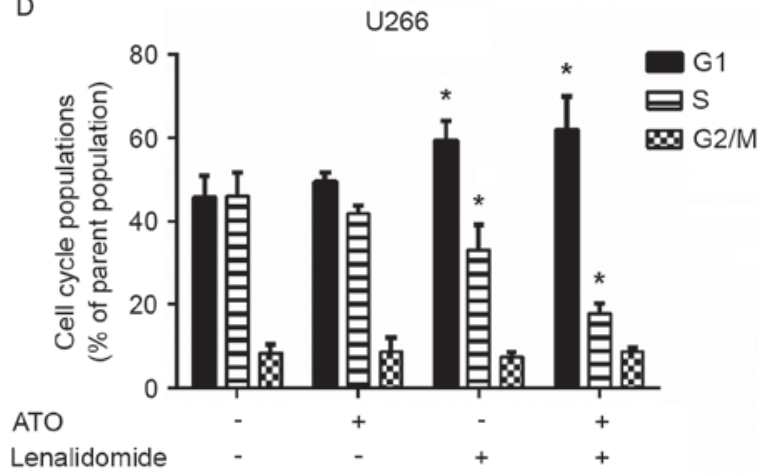

Figure 2. Combination of low doses of arsenic trioxide induces synergistic viability inhibition effect in MM cells. MM cell lines were pretreated with or without $0.1 \mu \mathrm{M}$ arsenic trioxide for $24 \mathrm{~h}$, and then treated with lenalidomide $(10 \mu \mathrm{M})$ (or vehicle) for another $24 \mathrm{~h}$, followed by: (A and B) Assessment of cell viability using Cell Counting kit-8; (C) assessment for apoptosis using annexin V-FITC/propidium iodide staining, followed by analysis on flow cytometry; (D) assessment of cell cycle populations through propidium iodide staining, followed by flow cytometry analysis. Experiments with single agents and the respective combinations were performed simultaneously. Values plotted are the mean \pm standard deviation $(\mathrm{n}=3)$. ${ }^{*} \mathrm{P}<0.05$ (compared with control group). MM, multiple myeloma; ATO, arsenic trioxide.

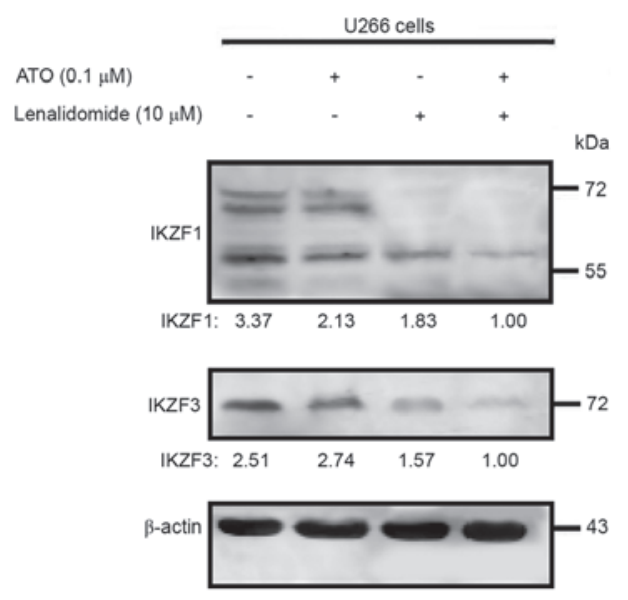

Figure 3. Effects of arsenic trioxide and lenalidomide on CRBN signaling. U266 cells were pretreated with $0.1 \mu \mathrm{M}$ arsenic trioxide (or vehicle equivalent) for $24 \mathrm{~h}$, and then incubated with $10 \mu \mathrm{M}$ lenalidomide was added for an additional $24 \mathrm{~h}$. Cells were harvested and IKZF1 and IKZF3, substrates of CRBN E3 ubiquitin ligase, were subjected to western blot analysis with anti-IKZF1 and -IKZF3 antibodies. Values indicate the fold-increase of protein levels following normalization to loading controls ( $\beta$-actin). CRBN, cereblon; ATO, arsenic trioxide; IKZF1, Ikaros family zinc finger 1.

In order to gain insights into the mechanism by which the decrease in cell viability of the combinations observed in the
CCK-8 assays, annexin V-FITC/propidium iodide apoptosis detection was conducted using flow cytometry (Fig. 2C). The combination treatment induced an apoptotic rate of $12.7 \%$, which was higher than those in the single agent group. The anti-proliferative activity of the combination treatment was further analyzed by evaluating the effects on the cell-cycle profile. The combination treatment of arsenic trioxide and lenalidomide induced an increase in the percentage of cells in G0/G1 phase, with a decrease in the percentage of those in proliferative $\mathrm{S}$ and $\mathrm{G} 2 / \mathrm{M}$ phases (Fig. 2D).

Arsenic trioxide plus lenalidomide downregulates IKZFI and IKZF3 expression levels. The ubiquitination and degradation of IKZF1 and IKZF3 (downstream proteins of CRBN) is necessary for the anti-myeloma effect of lenalidomide. As arsenic trioxide increased the CRBN expression level, whether the enhanced anti-MM effect of the combination therapy acted as the enhanced CRBN pathway was then examined. Western blot analysis revealed that arsenic trioxide as a single agent did not affect the expression level of IKZF1 or IKZF3 but, as anticipated, lenalidomide did downregulate expression of the two proteins. Multiple IKZF1 bands were detected, presumably due to alternative splicing (14). The use of arsenic trioxide and lenalidomide in combination had a significantly higher inhibitory effect on IKZF1 and IKZF3 protein expression 
levels, compared with the effect of either agent alone in the U266 cell line (Fig. 3), which could be evidence that arsenic trioxide can act as a sensitizer agent for the anti-myeloma agent lenalidomide.

\section{Discussion}

The present study revealed that low doses of arsenic trioxide potentiate the sensitivity of MM cell lines to lenalidomide by upregulating the expression of $\mathrm{CRBN}$, the anti-myeloma target of lenalidomide. The results of the present study demonstrated that low doses of arsenic trioxide upregulated CRBN mRNA level in U266 and RPMI8226 cell lines. In U266 cells, arsenic trioxide increased $\mathrm{CRBN}$ in doses lower than the $\mathrm{IC}_{25}$; a similar effect was also observed in RPMI8226 cells. CRBN protein expression levels validated the upregulation effect of low dose arsenic trioxide. Genetic heterogeneity is a hallmark of MM (15), which may explain, at least in part, the differences observed in the effects induced by arsenic trioxide between the two cell lines.

As the effect of elevated CRBN expression, whether the upregulation of CRBN caused by arsenic trioxide results in increased sensitivity of lenalidomide in MM cells was assessed. The results of the present study demonstrated that combined low doses of arsenic trioxide $(0.1 \mu \mathrm{M})$ and lenalidomide $(10 \mu \mathrm{M})$ triggered the inhibition of viability in U266 and RPMI8226 cells, which was only achievable at much higher doses of either agent alone. Apoptosis analysis also demonstrated the synergetic effect in the combination of these two drugs. Analysis of cell-cycle profiles confirmed that the combination therapy induced an increase in the percentage of cells in the G0/G1 phase, with a decrease in those in proliferative phases ( $\mathrm{S}$ and $\mathrm{G} 2 / \mathrm{M}$ phases), which could contribute to the increased inhibition of MM cell viability of this combination.

Previous studies found that the targets for proteasomal degradation ubiquitinated by lenalidomide-bound CRBN were identified as the B cell-specific transcription factors IKZF1 and IKZF3 $(14,16)$. Analysis of myeloma cell lines demonstrated that the loss of IKZF1 and IKZF3 expression is necessary and sufficient for the therapeutic effect of lenalidomide, through the downregulation of interferon regulatory factor 4 (IRF4), and this is associated with its anti-myeloma activity (14), suggesting a mechanism of action for lenalidomide on MM cells. Previous studies revealed that lenalidomide-bound CRBN acquires the ability to target IKZF1 and IKZF3 for proteasomal degradation, and analysis of myeloma cell lines demonstrating that the loss of IKZF1 and IKZF3 is necessary and sufficient for the therapeutic effect of lenalidomide $(14,16)$. The mechanism of the synergistic mechanism of these two drugs was assessed through detection of the expression levels of IKZF1 and IKZF3. The combination therapy of arsenic trioxide and lenalidomide had a significantly greater inhibitory effect on IKZF1 and IKZF3 protein expression levels, compared with the effect of either agent alone in the U266 cell line, whereas treatment with arsenic trioxide alone had no direct effect on the expression of these two proteins. These data are consistent with the findings of the present study, which found that arsenic trioxide increases the expression of CRBN in MM cells and the combination of low doses of arsenic trioxide and lenalidomide has a synergistic effect on decreasing viability rate, while increasing the apoptotic rate of MM cells. Myc proto-oncogene protein (MYC) is known to be a major downstream target of CRBN/IKZF1 signaling via IRF4 (7). Although MYC often acts to promote cell viability by driving entry into the cell cycle through the G1-S transition, the mechanism of G0/G1 cell cycle arrest induced by the combination treatment may be due to enhanced MYC downregulation, caused by the upstream downregulation of IKZF1 by lenalidomide.

The findings of the present study reveal a novel target for the traditional agent arsenic trioxide and provide the potential value of this drug in the treatment of the plasma cell disorder multiple myeloma. As the mechanism of the upregulation of CRBN by arsenic trioxide remains unclear, future studies are required to specify this mechanism, which could provide more comprehensive understanding to arsenic trioxide and investigate more therapeutic value. Since lenalidomide is increasingly used to treat $\mathrm{MM}$, the resistance to this IMiD is attracting a greater degree of attention. The findings of the present study potentially provide an efficient and economical way of working around resistance to lenalidomide. However, since lenalidomide acts more like an immunomodulatory drug, in vitro studies are not sufficient and in vivo data are required to assess the effect of the two drugs in combination in the future.

To summarize, the results of the present study demonstrate the ability of arsenic trioxide to increase expression of CRBN, the direct target of the anti-MM effect of lenalidomide, and the potent in vitro anti-MM activity of arsenic trioxide combined with lenalidomide at low doses. These findings provide the framework for the next step of in vivo studies and, if these studies are successful, for clinical trials of low-dose combination of arsenic trioxide and lenalidomide to increase response, overcome drug resistance, reduce side effects and improve patient outcome in MM.

\section{Acknowledgements}

The present study was supported by the National Natural Science Foundation of China (grant nos. 81172252 and 81500164), and the Capital of Clinical Characteristics and the Applied Research Fund of China (grant no. Z131107002213146).

\section{References}

1. Avigan D and Rosenblatt J: Current treatment for multiple myeloma. N Engl J Med 371: 961-962, 2014.

2. Kumar SK, Rajkumar SV, Dispenzieri A, Lacy MQ, Hayman SR, Buadi FK, Zeldenrust SR, Dingli D, Russell SJ, Lust JA, et al: Improved survival in multiple myeloma and the impact of novel therapies. Blood 111: 2516-2520, 2008.

3. Richardson PG, Blood E, Mitsiades CS, Jagannath S, Zeldenrust SR, Alsina M, Schlossman RL, Rajkumar SV, Desikan KR, Hideshima T, et al: A randomized phase 2 study of lenalidomide therapy for patients with relapsed or relapsed and refractory multiple myeloma. Blood 108: 3458-3464, 2006.

4. Kortuem KM, Zidich K, Schuster SR, Khan ML, JimenezZepeda VH, Mikhael JR, Fonseca R and Stewart AK: Activity of 129 single-agent drugs in 228 Phase I and II clinical trials in multiple myeloma. Clin Lymphoma Myeloma Leuk 14: 284-290.e5, 2014.

5. Quach H, Ritchie D, Stewart AK, Neeson P, Harrison S, Smyth MJ and Prince HM: Mechanism of action of immunomodulatory drugs (IMiDS) in multiple myeloma. Leukemia 24: 22-32, 2010. 
6. Ito T, Ando H, Suzuki T, Ogura T, Hotta K, Imamura Y, Yamaguchi $Y$ and Handa H: Identification of a primary target of thalidomide teratogenicity. Science 327: 1345-1350, 2010.

7. Zhu YX, Braggio E, Shi CX, Bruins LA, Schmidt JE, Van Wier S, Chang XB, Bjorklund CC, Fonseca R, Bergsagel PL, et al: Cereblon expression is required for the antimyeloma activity of lenalidomide and pomalidomide. Blood 118: 4771-4779, 2011.

8. Douer D and Tallman MS: Arsenic trioxide: New clinical experience with an old medication in hematologic malignancies. J Clin Oncol 23: 2396-2410, 2005.

9. Shen ZX, Chen GQ, Ni JH, Li XS, Xiong SM, Qiu QY, Zhu J, Tang W, Sun GL, Yang KQ, et al: Use of arsenic trioxide (As2O3) in the treatment of acute promyelocytic leukemia (APL): II. Clinical efficacy and pharmacokinetics in relapsed patients. Blood 89: 3354-3360, 1997.

10. Soignet SL, Maslak P, Wang ZG, Jhanwar S, Calleja E, Dardashti LJ, Corso D, DeBlasio A, Gabrilove J, Scheinberg DA, et al: Complete remission after treatment of acute promyelocytic leukemia with arsenic trioxide. N Engl J Med 339: 1341-1348, 1998.

11. Miller WH Jr, Schipper HM, Lee JS, Singer J and Waxman S: Mechanisms of action of arsenic trioxide. Cancer Res 62: $3893-$ 3903, 2002.
12. Chou TC: Theoretical basis, experimental design, and computerized simulation of synergism and antagonism in drug combination studies. Pharmacol Rev 58: 621-681, 2006.

13. Livak KJ and Schmittgen TD: Analysis of relative gene expression data using real-time quantitative PCR and the 2(-Delta Delta C(T)) method. Methods 25: 402-408, 2001.

14. Lu G, Middleton RE, Sun H, Naniong M, Ott CJ, Mitsiades CS, Wong KK, Bradner JE and Kaelin WG Jr: The myeloma drug lenalidomide promotes the cereblon-dependent destruction of Ikaros proteins. Science 343: 305-309, 2014.

15. Bergsagel PL and Kuehl WM: Molecular pathogenesis and a consequent classification of multiple myeloma. J Clin Oncol 23: 6333-6338, 2005

16. Krönke J, Udeshi ND, Narla A, Grauman P, Hurst SN, McConkey M, Svinkina T, Heckl D, Comer E, Li X, et al: Lenalidomide causes selective degradation of IKZF1 and IKZF3 in multiple myeloma cells. Science 343: 301-305, 2014. 